\title{
A Laser Interferometer Prototype with Pico-Meter Measurement Precision for Taiji Space Gravitational Wave Detection Mission in China
}

\author{
Yuqiong $\mathrm{Li}^{1} \cdot$ Chenyu Wang ${ }^{1,2} \cdot$ Luyu Wang ${ }^{3,4} \cdot$ Hang Liu ${ }^{1,2} \cdot$ Gang Jin ${ }^{1}$
}

Received: 3 April 2019 / Accepted: 18 November 2019

(C) Springer Nature B.V. 2020

\begin{abstract}
The laser interferometer is one of the most important key technologies for the space gravitational wave detection. A laser interferometer prototype with pico-meter measurement precision for Taiji mission in China is presented in the paper. The results showed that the path-length measurement precision reached $5 \mathrm{pm} / \sqrt{\mathrm{Hz}}$ within the frequency range of $10 \mathrm{mHz}-1 \mathrm{~Hz}$ by improving the temperature fluctuation noise and electronic readout noise of the laser interferometer, which meets the requirement of Taiji Pathfinder mission inside the frequency range of $1 \mathrm{mHz}-1 \mathrm{~Hz}$ under the weak-light condition. It would be a fine experimental platform for the key technologies demonstration including the laser pointing modulation and the laser phaselocking control to improve the precision for the low measurement noise.
\end{abstract}

Keywords Laser interferometer · Gravitational waves · Pico-meter measurement precision · Taiji

\section{Introduction}

In 2016, the LIGO (Laser Interferometer Gravitational-Wave Observatory) Scientific Collaboration and Virgo Collaboration observed gravitational wave (GW) from a binary black hole merger, and they demonstrated the true existence of GW several times during the next two years (LIGO Scientific Collaboration and Virgo Collaboration 2016a, b, 2017, 2018). To detect the GW of medium-low frequencies from milli-Hz to deci-Hz in which a space-based GW astronomy could complement its ground-based counterpart, a space mission called LISA (Laser Interferometer Space Antenna) mission was put forward by the

Yuqiong Li

liyuqiong@imech.ac.cn

1 National Microgravity Laboratory, Institute of Mechanics, Chinese Academy of Sciences, Beijing 100190, China

2 School of Engineering Science, University of Chinese Academy of Science, Beijing 100049, China

3 Center for LTA System Research and Development, Academy of Opto-electronics of Chinese Academy of Sciences, Beijing 10009, China

4 School of Optoelectronics, University of Chinese Academy of Science, Beijing 100049, China
ESA (European Space Agency), which could well avoid the limits of laser interferometer arm length and Earth seismic noise (Gair et al. 2013; Pitkin et al. 2011). The LISA is scheduled to be launched into space in 2034 as the ESA L3 mission (Vitale 2014; Sesana et al. 2014). To demonstrate the key technologies such as laser interferometer and drag-free control for the LISA mission, a LISA Pathfinder satellite was launched into space at the end of 2015. The results showed that the key technologies were better than the requirement of the LISA mission, including the laser interferometer reached femto-meter level and drag-free control achieved sub-femto-g free fall (Armano et al. 2016). DECIGO (DECi-heltz Gravitational-wave Observatory) is a Japanese space gravitational wave detector which is planned to be launched in 2030s (Musha et al. 2017). It consists of three drag-free satellites forming triangle shaped Fabry-Perot laser interferometer with the arm length of $1000 \mathrm{~km}$, whose strain sensitivity is designed to be $2 \times 10^{-24} / \sqrt{ } \mathrm{Hz}$ around $0.1 \mathrm{~Hz}$ (Sesana et al. 2014; Musha et al. 2017). The DECIGO working group have developed frequency and intensity stabilized lasers whose frequency noise is $d f<0.5 \mathrm{~Hz} / \sqrt{\mathrm{Hz}}$ and the intensity noise is $d I / I$ $<10^{-8} / \sqrt{\mathrm{Hz}}$ at the observation band around $1 \mathrm{~Hz}$ (Sesana et al. 2014; Suemasa et al. 2017a, b).

In China, a feasibility study of Space GW Detection has been launched by the CAS (Chinese Academy of Sciences) since 2008, meanwhile an investigation group in CAS for Space GW Detection was organized (Gong et al. 2011; Li 
et al. 2012, 2015). The Space GW Detection was listed in "2050 Development Project" of CAS in 2009, and the Space GW Detection Working Group in CAS was founded in 2012. In 2015, a Space GW Detection mission called Taiji was put forward by the CAS, which would consist of a triangle of three spacecraft in orbit around the Sun (Jin 2017; Cyranoski 2016). The Taiji's spacecraft would be separated by 3 million kilometers, and the required measurement sensitivity of laser interferometer would be $10 \mathrm{pm} / \sqrt{\mathrm{Hz}}$ within the frequency range of $0.1 \mathrm{mHz}-1 \mathrm{~Hz}$ (Li et al. 2018; $\mathrm{Hu}$ and $\mathrm{Wu} 2017$; Gong et al. 2015). The road map for Taiji mission could be summarized into three stages, as one of the most important steps, two Taiji Pathfinder satellites would be launched in 2025. The arm length between the two satellites would be set from $10,000 \mathrm{~km}$ to $100,000 \mathrm{~km}$, and the sensitivity will require $100 \mathrm{pm} / \sqrt{\mathrm{Hz}}$ within the frequency range of $0.1 \mathrm{mHz}-1 \mathrm{~Hz}$ (Liu et al. 2018). According to the design of the Taiji Pathfinder scheme, the weak-light and the maximum Doppler shift would be 50 $\mathrm{nW}$ and $20 \mathrm{MHz}$, respectively. There is another Chinese proposal called TianQin aims to detect the space gravitational waves in the millihertz frequencies $(0.1 \mathrm{mHz}-100 \mathrm{mHz})$, which is led by the Sun Yat-Sen University (Cyranoski 2016; Luo et al. 2016). TianQin is a constellation of three Earthorbiting spacecraft in a nearly equilateral triangle formation, of which the arm-length is $10^{5} \mathrm{~km}$ (Luo et al. 2016). The required measurement accuracy of laser interferometer is $1 \mathrm{pm} /$ $\sqrt{ } \mathrm{Hz} @ 6 \mathrm{mHz}$ (Luo et al. 2016). The working group have developed a dual-heterodyne laser interferometer for simultaneously measuring linear and angular displacements with resolutions of picometer and nanoradian, respectively (Yan et al. 2015).

As a major participant of the Taiji mission, the laser interferometer working group focuses on the research and development of key technologies, including the laser interferometer, phasemeter, laser phase-locking and laser-pointing etc. ( $\mathrm{Li}$ et al. 2012, 2015, 2018; Liu et al. 2014, 2015, 2018; Dong et al. 2014, 2015, 2016). In the previous research, an onground laser interferometer prototype has been constructed, of which the optical design is similar to the LISA Path-finder. The results showed that path-length measurement sensitivity reached $15 \mathrm{pm} / \sqrt{ } \mathrm{Hz}$ within the frequency regime of $0.1 \mathrm{~Hz}-$ $1 \mathrm{~Hz}$, and achieved $100 \mathrm{pm} / \sqrt{\mathrm{Hz}}$ inside the frequency regime of $1 \mathrm{mHz}-0.1 \mathrm{~Hz}$ (Luo et al. 2018) under the condition of milliwatt power of interference light. Recently, a new laser interferometer experimental system has been set up, of which the vacuum performance, vibration isolation and photodetector performance have been improved greatly. Under the condition that the interference light intensity is microwatt and nanowatt, the electronic readout and phasemeter measurement noise of the laser interferometer prototype is lower than $1 \mathrm{pm} /$ $\sqrt{\mathrm{Hz}}$ within the frequency regime of $9 \mathrm{mHz}-1 \mathrm{~Hz}$, and lower than $20 \mathrm{pm} / \sqrt{ } \mathrm{Hz}$ inside the frequency regime of $0.1 \mathrm{mHz}-9$ $\mathrm{mHz}$. The path-length measurement sensitivity reaches $5 \mathrm{pm} /$ $\sqrt{\mathrm{Hz}}$ within the frequency regime of $10 \mathrm{mHz}-1 \mathrm{~Hz}$, and better than $100 \mathrm{pm} / \sqrt{\mathrm{Hz}}$ inside the frequency regime of $1 \mathrm{mHz}-10$ $\mathrm{mHz}$, meeting the measurement sensitivity requirement of Taiji Pathfinder inside the frequency regime of $0.1 \mathrm{mHz}-$ $1 \mathrm{~Hz}$. This demonstrates that a laser interferometer prototype with pico-meter measurement precision during the long frequency regime has been realized, which is a step forward of the laser interferometer development.

\section{Design and Experimental Details}

\section{Description of the Experimental Setup}

Similar to the previous experimental setup, a clean-room of 1000 purification grade with $\pm 1{ }^{\circ} \mathrm{C}$ temperature adjustment precision was built ( $\mathrm{Li}$ et al. 2015). Inside the clean-room, an optical table with passive vibration isolation based on an isolated ground was adopted to avoid the vibration noise, and a vacuum chamber on the passive vibration isolation system was constructed to limit the thermal and electromagnetic noises. The vibration isolation system consists of an isolated ground and four suspended posts, the resonance frequency of which is lower than $1 \mathrm{~Hz}$. A rotary pump and a turbo pump were adopted to exhaust the air in the chamber. The lowest pressure of the vacuum chamber can reach $5 * 10^{-6} \mathrm{~Pa}$ while there is no any optical component inside it, and the lowest pressure can achieve $5^{*} 10^{-5} \mathrm{~Pa}$ while the optical bench and the laser interferometer are put inside it. When the rotary pump and turbo pump stopped, the pressure of the vacuum chamber can keep lower than $5 \times 10^{-2} \mathrm{~Pa}$ during the next $48 \mathrm{~h}$. The opening and closing of the vacuum chamber cover was driven by an elevator for the heavy weight. The schematic diagram and physical picture of the experimental setup are shown in Fig. 1 (a) and (b), respectively.

Similar to the previous design ( $\mathrm{Li}$ et al. 2015), the laser interferometer layout consists of a modulation bench and an optical bench, the schematic diagram is shown in Fig. 2. The modulation bench located outside of the vacuum chamber provides laser beam preparation. The linearly polarized light emitted from the laser (made in Beijing, wavelength $\lambda=$ $1064 \mathrm{~nm}$, power $P=300 \mathrm{~mW}$, frequency instability $\Delta f=$ $0.5 \mathrm{MHz} / \sqrt{\mathrm{Hz}}$ ) passes through a Faraday isolator to avoid back-reflection of the laser beam. Then, the laser frequency is modulated by using AOMs at approximately $100 \mathrm{MHz}$ with a frequency difference $f_{\text {het }}$ of $1 \mathrm{MHz}$, the relative frequency instability of which is $10^{-7}$. The role of the wedged plate is to make the laser beam emitted from the AOM's first diffraction become parallel to the optical bench. Here, the NDF is used to attenuate the light power, which can adjust four orders of magnitude continuously. Finally, the frequency-shifted beams are injected into the optical bench in the vacuum chamber by two polarization maintaining optical fibers. 


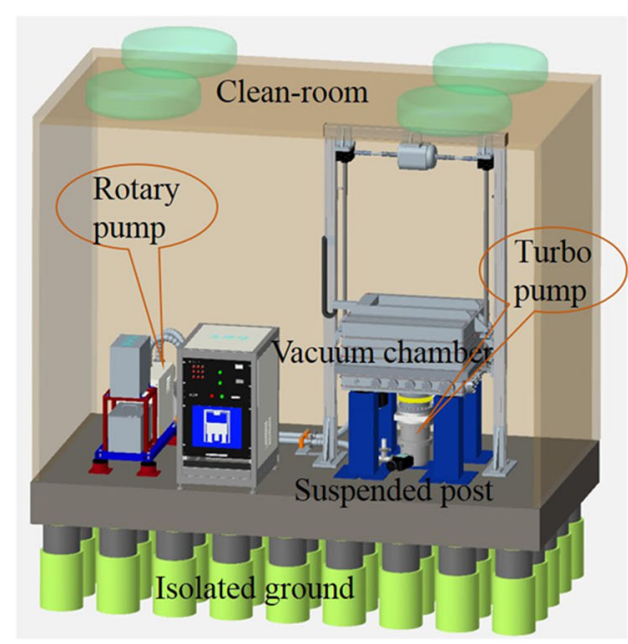

(a)

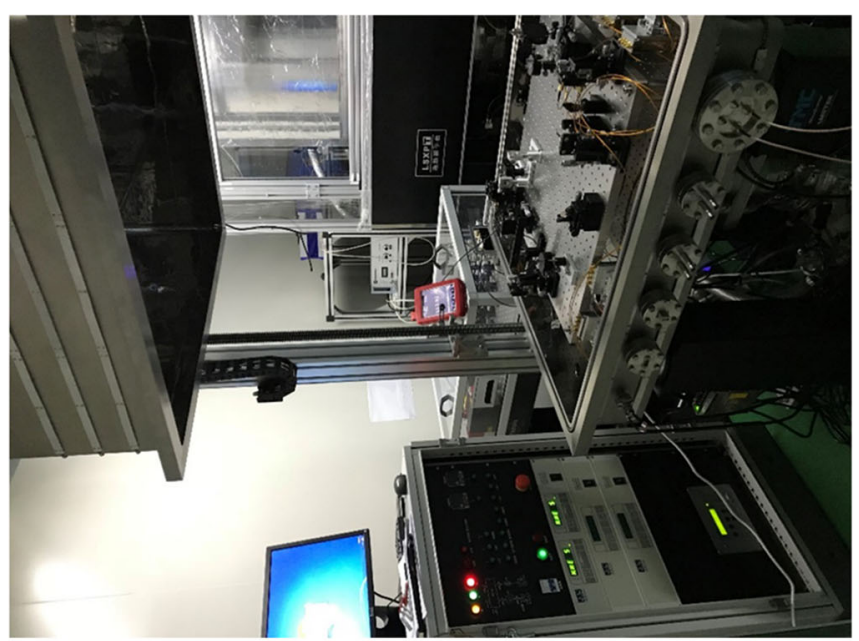

(b)

Fig. 1 Schematic diagram and physical picture of the experimental setup. a schematic diagram b physical picture

The optical bench located in the vacuum chamber contains two interferometers with equal arm-length, which performs the path-length fluctuations measurement. The previous investigations showed that the optical fiber had large temperature fluctuation noise (Luo et al. 2018), so a reference interferometer that senses the common-mode phase fluctuations is built to reduce the environmental noise, such as mechanical and thermal fluctuations, as shown in Fig. 3a. The measurement interferometer is sensitive to the relative distance of the two simulated testmasses (here replaced by two mirrors) to each other, as shown in Fig. 3b. Path-length fluctuations of the modulation bench resulting from the environmental noise are measured in each individual interferometer and canceled in the differential phase ' $M-R$ ' (' $M$ ' and ' $R$ ' refer to the measurement interferometer and the reference interferometer, respectively). So, path-length differences before the optical bench are canceled by referring all measurements to the reference interferometer, and only those on the optical bench are coupled into the path-length ranging. Here, PBS is adopted to splitter the laser light into P light and S light. In order to keep the light power of the two laser interferometers equal, two line polarizers are used to adjust the polarization state of the laser light.

\section{Photo-Detector}

In the future Taiji mission, the spacecraft would be separated by 3 million kilometers, but the laser power would be about $2 \mathrm{~W}$ for the consideration of life span. Meanwhile, only a small fraction of the transmitted laser beam can be received by the detector of

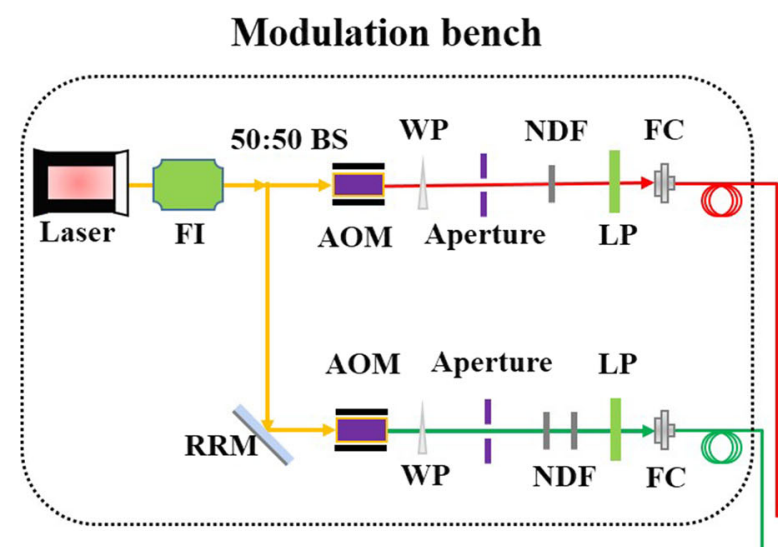

Fig. 2 Schematic diagram of the heterodyne interferometer. FI Faraday isolator, $\boldsymbol{B S}$ Beam splitter, $\boldsymbol{R} \boldsymbol{R M}$ Rectangular reflection mirror, $\boldsymbol{A O M}$ Acousto-optic modulator, WP Wedged prism, NDF Neutral density

\section{Optical bench}

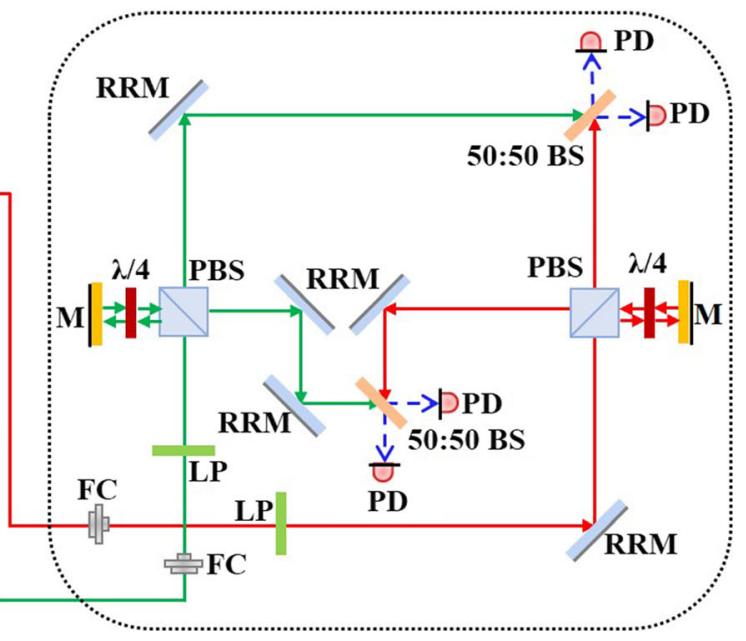

filter, $\boldsymbol{L P}$ Line polarizer, $\boldsymbol{F} \boldsymbol{C}$ Fiber coupler, $\boldsymbol{P} \boldsymbol{B} \boldsymbol{S}$ Polarizing beam splitter, $\boldsymbol{M}$ Mirror, $\boldsymbol{P D}$ Photo-detector 


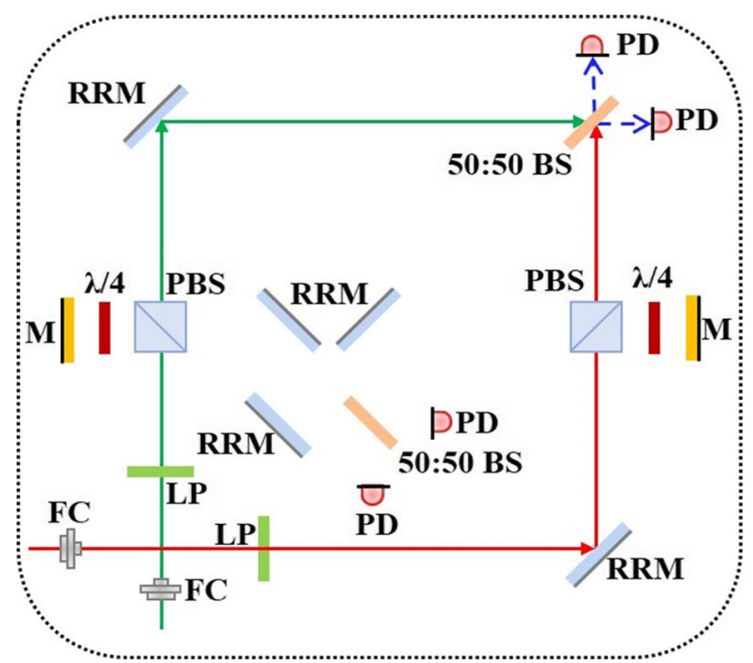

(a)

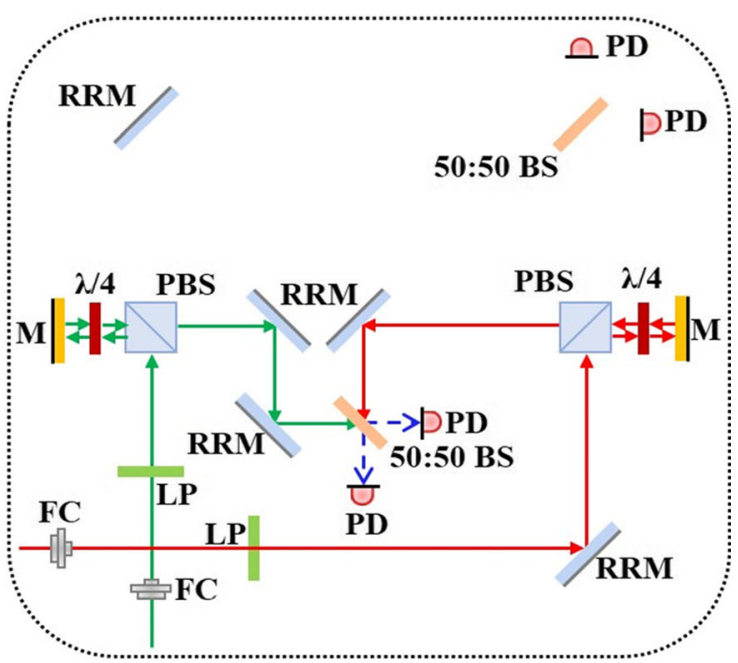

(b)

Fig. 3 Schematic diagram of the two interferometers. a the reference interferometer $\mathbf{b}$ the measurement interferometer. $\boldsymbol{F C}$ Fiber coupler, $\boldsymbol{L P}$ Line polarizer, $\boldsymbol{P B S}$ Polarizing beam splitter, $\boldsymbol{R} \boldsymbol{R} \boldsymbol{M}$ Rectangular reflection mirror, $\boldsymbol{M}$ Mirror, $\boldsymbol{B S}$ Beam splitter, $\boldsymbol{P D}$ Photo-detector

the remote satellite because of the large beam divergence and the finite aperture of the telescope. So, the received power on one of the detectors in the remote spacecraft would be $100 \mathrm{pW}$ approximately. In the previous investigations, the weak-light problem hasn't been solved for the low conversion gain of the photo-detector. In this experiment, the adopted photo-detector is developed by the Southwest Institute of Technical Physics in Chengdu, China, of which the schematic diagram is shown in Fig. 4. The power supply for the $\mathrm{V}_{\mathrm{CC}}$ and $\mathrm{V}_{\mathrm{DD}}$ is $12 \mathrm{~V}$ and $5 \mathrm{~V}$, respectively. Some key specifications are listed in the Table 1. According to the conversion gain, $100 \mathrm{pW}$ and $10 \mu \mathrm{W}$ interferometer lights can only produce $100 \mathrm{mV}$ of peak-to-peak voltage. In order to obtain better SNR (Signal-to-noise Ratio) signal, 10 $\mathrm{nW}$ and $10 \mu \mathrm{W}$ interferometer lights are adopted here. According to the design scheme of the Taiji Pathfinder, the received light power of the remote spacecraft is about $50 \mathrm{nW}$. So, the weak-light used here is lower than that of the Taiji Pathfinder. In particular, the NEP (Noise Equivalent Power) of the detector is greatly reduced by introducing a suitable capacitance into the photodetector chip.

\section{Phasemeter}

In the previous investigations, a digital phasemeter with multi-channel, which is built upon FPGA (Field

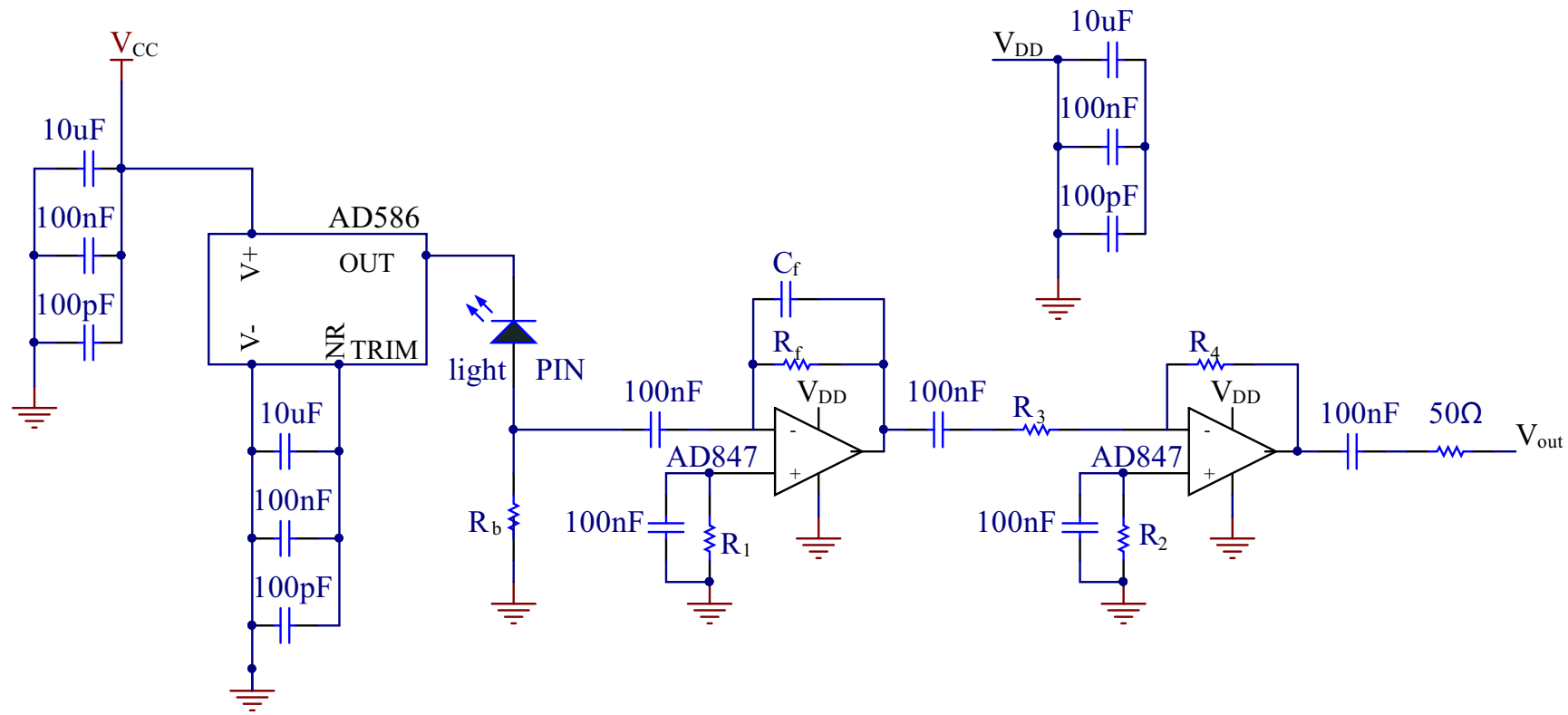

Fig. 4 Schematic diagram of the photo-detector 
Table 1 Key specifications of the photo-detector

\begin{tabular}{lllllr}
\hline $\begin{array}{l}\text { Active area } \\
\text { diameter }\end{array}$ & Quadrant & Responsivity & $\begin{array}{l}\text { Conversion } \\
\text { gain }\end{array}$ & $\begin{array}{l}\text { Noise equivalent } \\
\text { power }\end{array}$ & Bandwidth \\
\hline $0.5 \mathrm{~mm}$ & 1 & $0.68 \mathrm{~A} / \mathrm{W}$ & $3 \times 10^{6} \mathrm{~V} / \mathrm{W}$ & $4.5 \mathrm{pW} / \sqrt{\mathrm{Hz}}$ & $0-2 \mathrm{MHz}$ \\
\hline
\end{tabular}

Programmable Gate Array, TR4-530, Terasic) and based on DPLL (Digital Phase-locked Loop) scheme, has been developed by our group (Liu et al. 2014, 2015, 2018). Here the performance of the phasemeter was evaluated under the condition that the tested signal was generated by a functional generator (Agilent, 33522A), and the data were analyzed with a method called ASD (Amplitude Spectral Density) and LASD (Linearization Amplitude Spectral Density), the toolbox of which was developed by the Max Planck Institute for Gravitational Physics. The frequency and amplitude of the tested signal are $1 \mathrm{MHz}$ and $800 \mathrm{mV}$, respectively. For decreasing the influence of phase error between different signals, the noise spectrum is tested in the condition of the zero measurement which the signal from the functional generator is split into two and then delivered into the two channels of the phasemeter (Liu et al. 2018). The results are shown in Fig. 5, from which can be concluded that the phase measurement sensitivity can satisfy the requirement of Taiji mission in the frequency ranges between $9 \mathrm{mHz}$ and $1 \mathrm{~Hz}, 0.1 \mathrm{mHz}$ and $3 \mathrm{mHz}$. However, the analog frontend noise caused by the thermal drift, sampling noise and frequency jitter noise results in the sensitivity doesn't meet the requirement within the frequency regime of $3 \mathrm{mHz}-9 \mathrm{mHz}$ (Liu et al. 2015, 2018). Compared to the previous results, the measurement sensitivity of the phasemeter is improved for adopting a Ultra-Stable Oscillator (USO, the time stability and accuracy are $10^{-12}$ and $5 \times 10^{-11}$, respectively, in the time range of $1-10,000 \mathrm{~s}$ ) instead of an ordinary oscillator to drive it. For the ADC is driven by local oscillator, when sampling the signal with ADC, the frequency variance of the USO will introduce phase noise into the sampled signal. This noise is called sampling jitter noise:

$\delta_{\varphi}=2 \pi \times \delta_{t} \times f_{b}$

where $\delta_{t}$ is the time stability of the USO, and $f_{b}$ is the frequency of tested signal. For a $1 \mathrm{MHz}$ tested signal, the absolute value of time jitter noise is $2 \pi \times 10^{-6} \mathrm{rad}$. Meanwhile, the testing environment has more stable temperature, so the electronic noise caused by the Brownian movement of the electron reduced a lot (Liu et al. 2018).

\section{Results and Discussion}

Before evaluating the path-length measurement sensitivity of the prototype, it is necessary to measure the electronic readout and phasemeter measurement noise of the laser interferometer prototype. The test program is as follows: dividing one of the interferometer's readout from one photodiode into two parts, then adopting the phasemeter to measure the phase difference between them. The ASD and LASD curves in Fig. 6 show that the electronic readout and phasemeter measurement noise of the prototype is lower than $1 \mathrm{pm} / \sqrt{ } \mathrm{Hz}$ inside the frequency regime of $9 \mathrm{mHz}-1 \mathrm{~Hz}$, but increasing with a linear function with
Fig. 5 Phase measurement noise of the phasemeter

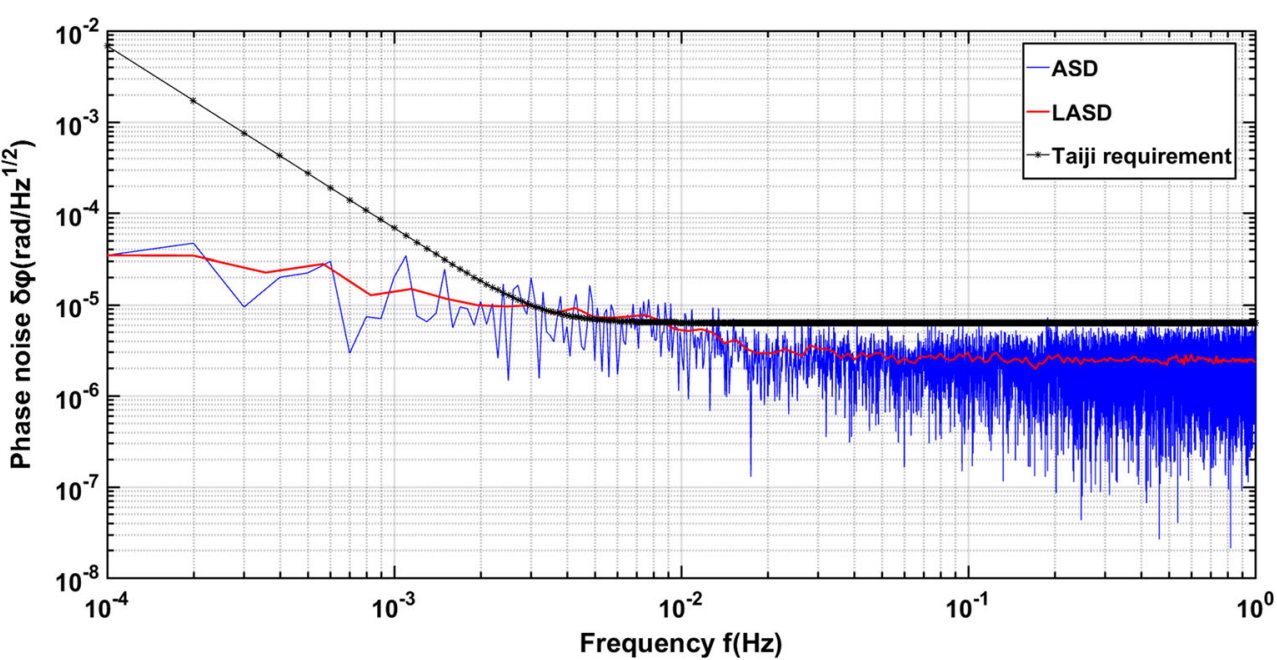


Fig. 6 The electronic readout and phasemeter noise of the laser interferometer prototype

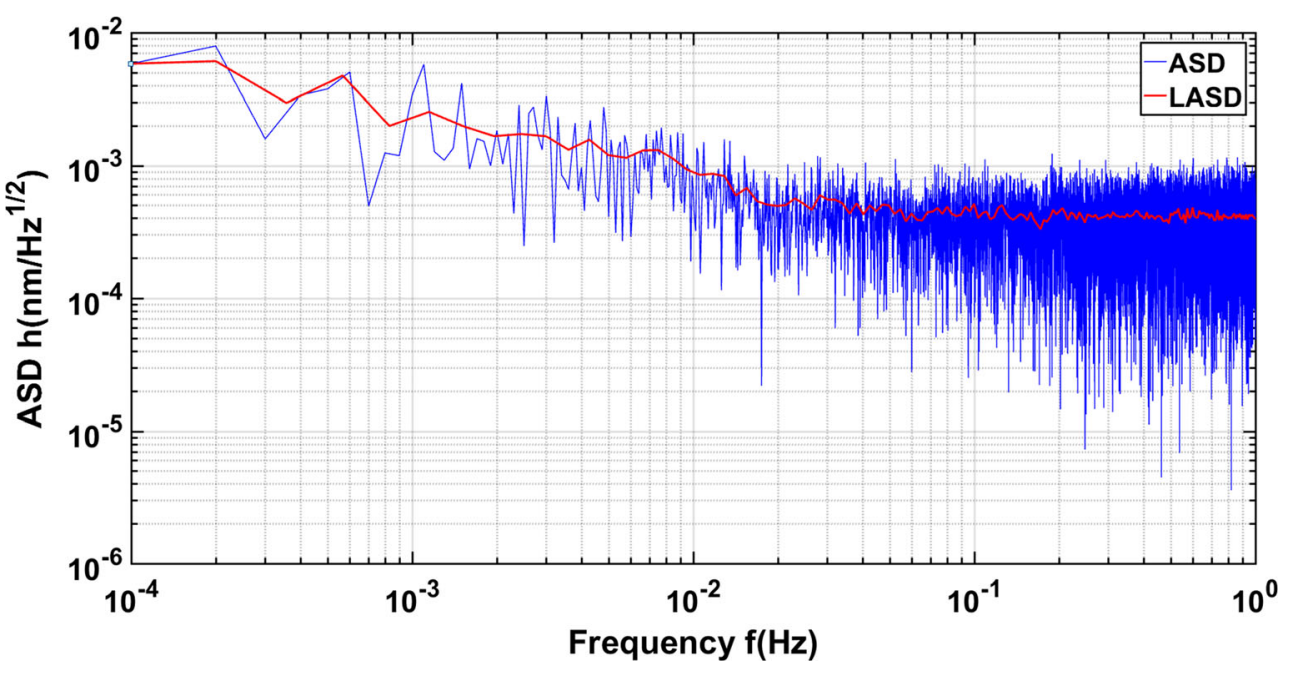

decreasing frequency within the frequency range of $0.1 \mathrm{mHz}-40 \mathrm{mHz}$. However, it is still lower than $6 \mathrm{pm} / \sqrt{ } \mathrm{Hz}$. Compared to the previous investigations, the noise has reduced largely for the SNR of the photodetector has been improved greatly (Dehne et al. 2012; Li et al. 2015).

The path-length measurement sensitivity of the measurement interferometer is shown in the Fig.7. From it can be known that the sensitivity is about $5 \mathrm{pm} / \sqrt{ } \mathrm{Hz}$ in the frequency range of $10 \mathrm{mHz}$ and $1 \mathrm{~Hz}$, and decreasing with a linear function with decreasing frequency within the frequency range of $0.1 \mathrm{mHz}-10 \mathrm{mHz}$. It also can be seen that the sensitivity meets the requirement of the Taiji Pathfinder, but still there is a gap compared to the requirement of the Taiji mission. From the comparisons between the electronic readout and phasemeter measurement noise and the measurement sensitivity of the laser interferometer can be concluded that the following techniques should be improved for meeting the measurement sensitivity requirement of the Taiji mission: (1) the frequency stability of the laser, the frequency instability $\Delta f$ of the laser adopted in this experiment is about $0.5 \mathrm{MHz} / \sqrt{ } \mathrm{Hz}$, which results in a large frequency noise $\delta L=(\Delta f / f) \Delta L=3.33 \mathrm{pm} / \sqrt{\mathrm{Hz}}(f$ is the frequency of the laser, the optical path-length difference $\Delta L$ is evaluated to be $2 \mathrm{~mm}$ ) ( $\mathrm{Li}$ et al. 2015). This is the main noise affecting the measurement sensitivity. So, a more stable frequency laser $(\Delta f=1 \mathrm{kHz} / \sqrt{\mathrm{Hz}})$ should be adopted in the future; (2) the mounting means of the optics, the mounting brackets of all components made of aluminum are used to install the optics in this experiment, and the thermal expansion coefficient of the brackets is higher than $10^{-6} /{ }^{\circ} \mathrm{C}$. This will not only bring large thermal noise into the laser interferometer, but also not be suitable for the flying mode in the future. The hydroxycatalysis technique is the most acceptable way for
Fig. 7 Path-length measurement sensitivity of the laser interferometer prototype

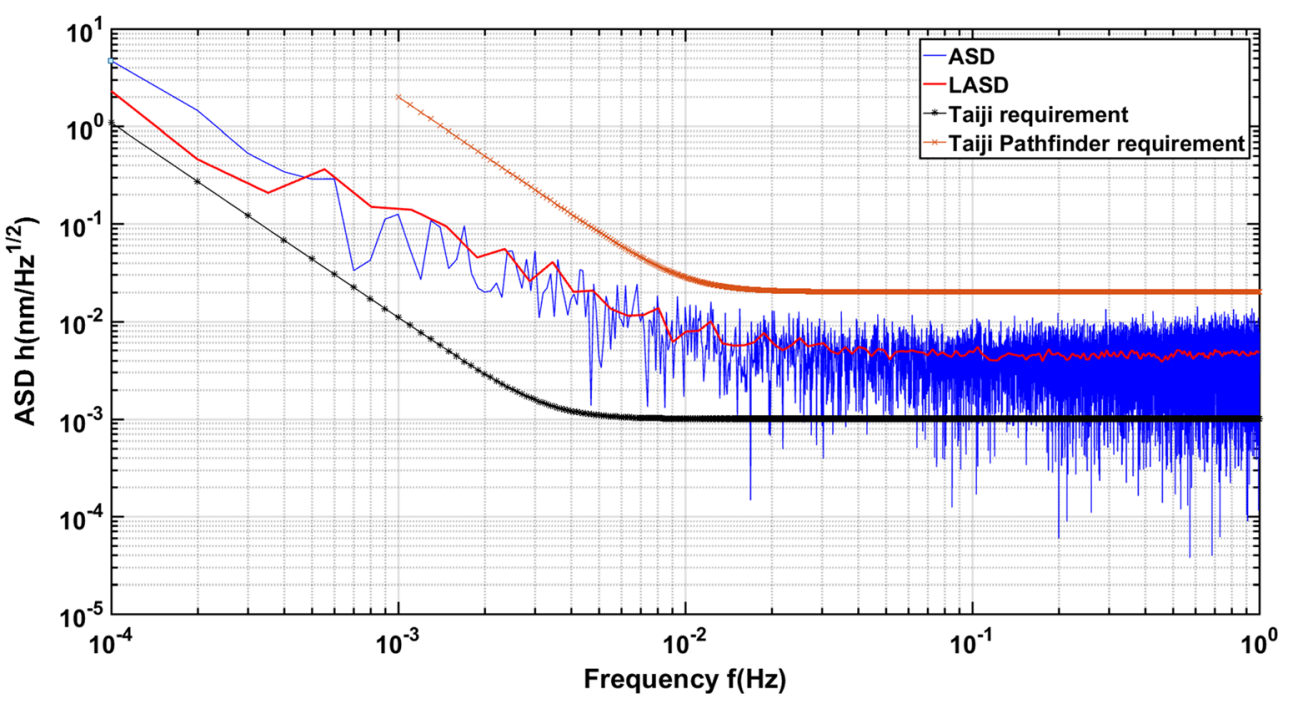


improving the fixing, which will be taken into account during the next step; (3) the analog-to-digital converter of the phasemeter, the requirement for the peak-to-peak voltage of the input signal is higher than $300 \mathrm{mV}$, which is a huge challenge for $100 \mathrm{pW}$ weak-light level in the future; (4) the conversion gain and bandwidth of the photodetector, the trade-off between the conversion gain and bandwidth should be considered for the weaklight is only $100 \mathrm{pW}$ and Doppler shift can reach $25 \mathrm{MHz}$ in the future Taiji mission; (5) the shot noise, which can be calculated by the following formula (McNamara 2005):

$\delta \varphi=\sqrt{\frac{e}{R \eta P}}$,

where $e$ is the electron charge $\left(1.6 \times 10^{-19} \mathrm{C}\right)$, and $R$ is the responsivity of the photodetector $(0.68 \mathrm{~A} / \mathrm{W})$. If the heterodyne efficiency $\eta$ is approximately $80 \%$, and the weak-light power $\mathrm{P}$ is $10 \mathrm{nW}$. The shot noise is $5.42 \mu \mathrm{rad} / \sqrt{\mathrm{Hz}}$, e.g. less than $1 \mathrm{pm} / \sqrt{\mathrm{Hz}}$. But when the light power lowers down to $100 \mathrm{pW}$, the shot noise will reach $54.2 \mu \mathrm{rad} / \sqrt{\mathrm{Hz}}$, nearly $10 \mathrm{pm} / \sqrt{ } \mathrm{Hz}$.

The noises of the laser interferometer prototype have been analyzed in the previous investigations ( $\mathrm{Li}$ et al. 2015), which won't be elaborated detailedly here. In this experiment, the noise is reduced mainly due to the suppression of the thermal noise and the electronic noise [Dehne et al. 2012; Li et al. 2015]. The pressure of vacuum chamber reduces from $1 \mathrm{~Pa}$ to $5^{*} 10^{-2} \mathrm{~Pa}$ and the NEP of the photodetector lowers from $77 \mathrm{pW} / \sqrt{\mathrm{Hz}}$ to $4.5 \mathrm{pW} / \sqrt{\mathrm{Hz}}$. In the previous investigations, the precision of laser phase-locking and laser pointing is limited by the thermal noise and electronic noise of the laser interferometer (Dong et al. 2014, 2015, 2016). Now, the path-length measurement sensitivity is better than $10 \mathrm{pm} / \sqrt{\mathrm{Hz}}$, which shows that the thermal noise and electronic noise of the laser interferometer have been restrained well. Under this noise level, many other noises could be observed, and the precision of laser phaselocking, laser pointing and other key technologies could also be improved greatly.

\section{Conclusions and Outlook}

Some results of a laser interferometer prototype with picometer measurement precision for Taiji mission in China were presented in the paper. The results showed that the path-length measurement precision reached $5 \mathrm{pm} / \sqrt{ } \mathrm{Hz}$ within the frequency range of $10 \mathrm{mHz}-1 \mathrm{~Hz}$, meeting the requirement of the Taiji Pathfinder mission inside the frequency range of $1 \mathrm{mHz}$ $-1 \mathrm{~Hz}$ under the weak-light condition. It would be a fine experimental platform for the laser pointing modulation and laser phase-locking control to improve the precision. But this is just an on-ground laser interferometer demonstration. Next step, a more stable frequency laser will be adopted, and the gain and bandwidth of the photodetector will be improved to observe the shot noise limit. Meanwhile, using hydroxycatalysis surface bonding techniques to fix the optics on an ultra-stable glassceramic baseplate made of Clearceram or Zerodur is also the main work for the laser interferometer.

Acknowledgements Authors thank the Southwest Institute of Technical Physics in Chengdu for providing the photodetector. This work is supported by the Strategic Priority Research Program of the Chinese Academy of Sciences, Grant No. XDB23030200, and the National Natural Science Foundation of China, Grant No. 61575209, and the Youth Innovation Promotion Association, Chinese Academy of Sciences, Grant No. 2018024.

\section{References}

Armano, M., Audley, H., Auger, G., Baird, J.T., Bassan, M., Binetruy, P., Born, M., Bortoluzzi, D., Brandt, N., Caleno, M., Carbone, L., Cavalleri, A., Cesarini, A., Ciani, G., Congedo, G., Cruise, A.M., Danzmann, K., de Deus Silva, M., De Rosa, R., Diaz-Aguiló, M., Di Fiore, L., Diepholz, I., Dixon, G., Dolesi, R., Dunbar, N., Ferraioli, L., Ferroni, V., Fichter, W., Fitzsimons, E.D., Flatscher, R., Freschi, M., García Marín, A.F., García Marirrodriga, C., Gerndt, R., Gesa, L., Gibert, F., Giardini, D., Giusteri, R., Guzmán, F., Grado, A., Grimani, C., Grynagier, A., Grzymisch, J., Harrison, I., Heinzel, G., Hewitson, M., Hollington, D., Hoyland, D., Hueller, M., Inchauspí, H., Jennrich, O., Jetzer, P., Johann, U., Johlander, B., Karnesis, N., Kaune, B., Korsakova, N., Killow, C.J., Lobo, J.A., Lloro, I., Liu, L., López-Zaragoza, J.P., Maarschalkerweerd, R., Mance, D., Martín, V., Martin-Polo, L., Martino, J., MartinPorqueras, F., Madden, S., Mateos, I., McNamara, P.W., Mendes, J., Mendes, L., Monsky, A., Nicolodi, D., Nofrarias, M., Paczkowski, S., Perreur-Lloyd, M., Petiteau, A., Pivato, P., Plagnol, E., Prat, P., Ragnit, U., Raïs, B., Ramos-Castro, J., Reiche, J., Robertson, D.I., Rozemeijer, H., Rivas, F., Russano, G., Sanjuán, J., Sarra, P., Schleicher, A., Shaul, D., Slutsky, J., Sopuerta, C.F., Stanga, R., Steier, F., Sumner, T., Texier, D., Thorpe, J.I., Trenkel, C., Tröbs, M., Tu, H.B., Vetrugno, D., Vitale, S., Wand, V., Wanner, G., Ward, H., Warren, C., Wass, P.J., Wealthy, D., Weber, W.J., Wissel, L., Wittchen, A., Zambotti, A., Zanoni, C., Ziegler, T., Zweifel, P.: Sub-Femto- g Free Fall for Space-Based Gravitational Wave Observatories: LISA Pathfinder Results. Phys. Rev. Lett. 116(23), 231101 (2016)

Cyranoski, D.: Chinese gravitational-wave hunt hits crunch time. Nature. 531(7593), 150-151 (2016)

Dehne, M., Tröbs, M., Heinzel, G., Danzmann, K.: Verification of polarising optics for the LISA optical bench. Opt. Express. 20(25), 27274-27287 (2012)

Dong, Y.H., Liu, H.S., Luo, Z.R., Li, Y.Q., Jin, G.: Methodological demonstration of laser beam pointing control for space gravitational wave detection missions. Rev. Sci. Instrum. 85(7), 074501 (2014)

Dong, Y.H., Liu, H.S., Luo, Z.R., Li, Y.Q., Jin, G.: Principle demonstration of fine pointing control system for inter-satellite laser communication. Sci. China Tech. Sci. 58(3), 449-453 (2015)

Dong, Y.H., Liu, H.S., Luo, Z.R., Li, Y.Q., Jin, G.: A comprehensive simulation of weak-light phase-locking for space-borne gravitational wave antenna. Sci. China Tech. Sci. 59(5), 730-737 (2016) 
Gair, J.R., Vallisneri, M., Larson, S.L., Baker, J.G.: Testing general relativity with low-frequency. Space-Based Gravitational-Wave Detectors. Living Rev. Relativ. 16(1), 7 (2013)

Gong, X.F., Xu, S.N., Bai, S., Cao, Z.J., Chen, G.R., Chen, Y.B., He, X.K., Heinzel, G., Lau, Y.K., Liu, C.Z., Luo, J., Luo, Z.R., Paton, A.P., Ruediger, A., Shao, M.X., Spurzem, R., Wang, Y., Xu, P., Yeh, H.C., Yuan, Y.Y., Zhou, Z.B.: A scientific case study of an advanced LISA mission. Class. Quantum Grav. 28, 094012 (2011)

Gong, X.F., Lau, Y.K., Xu, S.N., Seoane, P.A., Bai, S., Bian, X., Cao, Z.J., Chen, G., Chen, X., Ding, Y.W., Dong, P., Gao, W., Heinzel, G., Li, M., Li, S., Liu, F.K., Luo, Z.R., Shao, M.X., Spurzem, R., Sun, B.S., Tang, W.L., Wang, Y., Xu, P., Yu, P., Yuan, Y.F., Zhang, X.M., Zhou, Z.B.: Descope of the ALIA mission. IOP Conf. Series: Journal of Physics: Conf. Series. 610, 012011 (2015)

Hu, W.R., Wu, Y.L.: The Taiji program in space for gravitational wave physics and the nature of gravity. Natl. Sci. Rev. 4(5), 685-686 (2017)

Jin, G.: Program in space detection of gravitational wave in Chinese Academy of Sciences IOP Conf. Series: Journal of Physics: Conf. Series. 840, 012009 (2017)

Li, Y.Q., Luo, Z.R., Liu, H.S., Dong, Y.H., Jin, G.: Laser interferometer used for satellite-satellite tracking: an on-ground methodological demonstration. Chin. Phys. Lett. 29(7), 79501 (2012)

Li, Y.Q., Luo, Z.R., Liu, H.S., Dong, Y.H., Jin, G.: Path-length measurement performance evaluation of polarizing laser interferometer prototype. Appl. Phys. B Lasers Opt. 118(2), 309-317 (2015)

Li, Y.Q., Luo, Z.R., Liu, H.S., Gao, R.H., Jin, G.: Laser interferometer for space gravitational waves detection and earth gravity mapping. Microgravity Sci. Technol. 30(6), 817-829 (2018)

LIGO Scientific Collaboration and Virgo Collaboration: GW151226: Observation of Gravitational Waves from a 22-Solar-Mass Binary Black Hole Coalescence. Phys. Rev. Lett. 116(24), 241103 (2016a)

LIGO Scientific Collaboration and Virgo Collaboration: Observation of Gravitational Waves from a Binary Black Hole Merger. Phys. Rev. Lett. 116(6), 061102 (2016b)

LIGO Scientific Collaboration and Virgo Collaboration: GW170104: Observation of a 50-Solar-Mass Binary Black Hole Coalescence at Redshift 0.2. Phys. Rev. Lett. 118(22), 221101 (2017)

LIGO Scientific Collaboration and Virgo Collaboration: GW170817: Implications for the Stochastic Gravitational-Wave Background from Compact Binary Coalescences. Phys. Rev. Lett. 120(9), 091101 (2018)

Liu, H.S., Dong, Y.H., Li, Y.Q., Luo, Z.R., Jin, G.: The evaluation of phasemeter prototype performance for the space gravitational waves detection. Rev. Sci. Instrum. 85(2), 024503 (2014)

Liu, H.S., Dong, Y.H., Luo, Z.R., Li, Y.Q., Jin, G.: Multi-channel phasemeter and its application in the heterodyne laser interferometry. Sci. China Tech. Sci. 58(4), 746-749 (2015)

Liu, H.S., Luo, Z.R., Jin, G.: The development of Phasemeter for Taiji space gravitational wave detection. Microgravity Sci. Technol. 30(6), 775-781 (2018)
Luo, J., Chen, L.S., Duan, H.Z., Gong, Y.G., Hu, S.C., Ji, J.H., Liu, Q., Mei, J.W., Milyukov, V., Sazhin, M., Shao, C.G., Toth, V.T., Tu, H.B., Wang, Y.M., Wang, Y., Yeh, H.C., Zhan, M.S., Zhang, Y.H., Zharov, V., Zhou, Z.B.: TianQin: a space-borne gravitational wave detector. Class. Quantum Grav. 33(3), 035010 (2016)

Luo, Z.R., Liu, H.S., Jin, G.: The recent development of interferometer prototype for Chinese gravitational wave detection pathfinder mission. Opt. Laser Technol. 105, 146-151 (2018)

McNamara, P.W.: Weak-light phase locking for LISA. Class. Quantum Grav. 22(10), S243-S247 (2005)

Musha, M., DECIGO Collaborations: Japanese space gravitational wave antenna DECIGO and DPF. Society of Photo-optical Instrumentation Engineers Society of Photo-Optical Instrumentation Engineers (SPIE) Conference Series (2017)

Pitkin, M., Reid, S., Rowan, S., Hough, J.: Gravitational wave detection by interferometry (ground and space). Living Rev. Relativ. 14(5), 75 (2011)

Sesana, A., Weber, W.J., Killow, C.J., Perreur-Lloyd, M., Robertson, D.I., Ward, H., Fitzsimons, E.D., Bryant, J., Cruise, A.M., Dixon, G., Hoyland, D., Smith, D., Bogenstahl, J., McNamara, P.W., Gerndt, R., Flatscher, R., Hechenblaikner, G., Hewitson, M., Gerberding, O., Barke, S., Brause, N., Bykov, I., Danzmann, K., Enggaard, A., Gianolio, A., Vendt Hansen, T., Heinzel, G., Hornstrup, A., Jennrich, O., Kullmann, J., Møller-Pedersen, S., Rasmussen, T., Reiche, J., Sodnik, Z., Suess, M., Armano, M., Sumner, T., Bender, P.L., Akutsu, T., DECIGO working group, Sathyaprakash, B.S.: Space-based detectors. Gen. Relativ. Gravit. 46(12), 1793 (2014)

Suemasa A., Shimo-oku A., Nakagawa K., Musha M.: Developments of highly frequency and intensity stabilized lasers for space gravitational wave detector: DECIGO. 2017 Conference on Lasers and ElectroOptics Europe \& European Quantum Electronics Conference (CLEO/Europe-EQEC) Society of Photo-Optical Instrumentation Engineers (SPIE) Conference Series, 2017a

Suemasa, A., Nakagawa K., Musha M.: Highly frequency-stabilized laser for space gravitational wave detector DECIGO/DPF. International Conference on Space Optics 2014 Society of Photo-Optical Instrumentation Engineers (SPIE) Conference Series, 2017b

Vitale, S.: Space-borne gravitational wave observatories. Gen. Relativ. Gravit. 46(5), 1730 (2014)

Yan, H., Duan, H.Z., Li, L.T., Liang, Y.R., Luo, J., Yeh, H.C.: A dualheterodyne laser interferometer for simultaneous measurement of linear and angular displacements. Rev. Sci. Instrum. 86(12), $123102(2015)$

Publisher's Note Springer Nature remains neutral with regard to jurisdictional claims in published maps and institutional affiliations. 